University of Nebraska - Lincoln

DigitalCommons@University of Nebraska - Lincoln

French Language and Literature Papers

Modern Languages and Literatures, Department

2021

\title{
À propos de Lydie Salvayre
}

Mahir Guven

Follow this and additional works at: https://digitalcommons.unl.edu/modlangfrench

Part of the French and Francophone Language and Literature Commons

This Article is brought to you for free and open access by the Modern Languages and Literatures, Department of at DigitalCommons@University of Nebraska - Lincoln. It has been accepted for inclusion in French Language and Literature Papers by an authorized administrator of DigitalCommons@University of Nebraska - Lincoln. 


\section{À propos de Lydie Salvayre}

Cher Professeur Notte,

Vous noterez que je continue à buter sur votre patronyme. Allez savoir pourquoi ma mémoire n'a pas enregistré Motte. Allez comprendre le fonctionne du cerveau. Vous en savez quelque chose vous? Moi non ? Il faudrait demander à Lydie Salvayre. Vous ne pensez pas ? La fille a exercé comme psychiatre. La tronche, la caboche, elle connaît, pendant plus de trente ans, elle a nagé dans les délires et les névroses. Et ? Donc? De quoi je parle? J'y viens, cher Professeur. Patience. Faisons à la Française. D'abord, les politesses.

Un grand, un immense, un interstellaire merci, cher Professeur pour l'honneur que vous me faites en me proposant de rédiger un article de 2.000 mots « Sur l'écriture de Lydie Salvayre ». Moi petit homme de trente-deux ans qui sait à peine écrire. Qui avait à peine lu Lydie Salvayre avant votre proposition. Juste son Pas pleurer, parce que Goncourt, surtout parce que Guerre d'Espagne, aussi parce que parents réfugiés, parce que moi avoir histoire similaire, mais d'un autre pays, à une autre époque, et parce que dans la lecture, on cherche aussi un peu de soi-même. J'ai en mémoire de m'être senti en terrain connu dès les premières pages.

Published in Lydie Salvayre, maintenant même, ed. Warren Motte (Lincoln, NE : Zea Books, 2021). doi: 10.32873/unl.dc.zea.1289 
Quelque part comme chez moi, par le rapport à la mère, à la langue, au déracinement, à l'amour de la littérature, mais chut ! Je vais y revenir. Quatre ans plus tard, grâce à votre cadeau divin, à votre offre du diable, à votre proposition que je ne pouvais pas refuser, me voici à tenter de commander chez une libraire allemande d'Hambourg, une grande partie de l'œuvre de Lydie Salvayre. Lydie was? Was haben Sie gesagt? Salvaro ? Kenne ich nicht ... Sie hat ein Goncourt gewonnen! Oh, wie schön!

Pourtant Madame la libraire allemande du Bücherstube de la Rothenbaumchaussee à Hambourg, la Docteure Salvayre est l'une des voix majeures de la littérature française contemporaine. Elle pèse sur le game, comme diraient les jeunes. On l'invite sur les plateaux télé pour parler des livres des autres. D'après elle, elle s'en sort mieux que pour ses propres livres, parce que selon elle, elle se débrouille mal à l'oral. Faux Docteure Salvayre, laissez votre modestie tranquille au chaud dans votre aire de Broca. À l'inverse de l'essentiel des Français, la Docteure Salvayre parle comme elle écrit, c'est précis, juste, économe, sans faute, elle ne snobe jamais, flatte l'intelligence, n'envoie pas cinquantedouze références au visage pour démontrer son art, mais juste quelques-unes, utiles à sa narration, qui permettent de s'érudir. S'érudir? Je viens de chercher le mot. Introuvable. Je viens de lire sur le forum de languefrançaise.net que les dictionnaires français ne l'auraient pas référencé.

Constatez par vous-même. 
Sujet : Un mot pour désigner le fait de devenir plus intelligent, plus cultivé.

par Abouricha

Réponse de papy à 18-09-2010 à 00:08

« Cher Abouricha, j'ai pensé à s'intelligenter. Mais le mot fait un peu trop barbare à l'oreille, on sentirait tout de suite que c'est une invention de mon cru.

Le verbe s'érudir que DB propose est pourtant très intéressant, il convient tout à fait à ce que vous cherchez, mais quel dommage que le mot n'existe pas ».

S'érudir donc. Notez-le cher Professeur. Il est pas mal ce mot non ? Vous me demandiez comment écrit Lydie Salvayre, je vous sers une réponse sur un plateau. Elle invente des mots, des trouvailles, des perles compréhensibles par elles-mêmes ou par le sens de la phrase. Lire Lydie, c'est autant voyager dans la langue que par le récit. Sans me lancer dans une psychanalyse littéraire, pour laquelle le talent me manque davantage encore que pour la critique, je dirais, en comparant la vie du Docteure Salvayre à la mienne, que son goût immodéré et insolent pour le tripotage des mots provient de sa double culture. Cela doit venir de la madre. De sa daronne. Ouvrez Pas pleurer et enjaillez-vous à découvrir Madame Montse Arjona, sa mère, faire danser le hip-hop à la langue française sur un fond de flamenco. Maintenant, prenez une respiration, réfléchissez, vous êtes enfant, dans les années cinquante, dix ans après une terrible guerre, dans un pays en construction, vous allez à l'école de la République française, bien élitiste, où l'on vous chahute les oreilles, et même les doigts, si vous accordez un participe passé avec le verbe avoir. De retour à la maison, votre madre, votre maman, votre mère, votre matrice, la source, l'origine de votre 
vie, vous chérit dans un français estropié, bassiné d'espagnol, tout en vous sermonnant d'étudier avec sérieux. Vous êtes balancé dans un canyon entre deux cultures. Plus tard, vous en riez. Et pour la vie, vous nagerez d'une rive à l'autre. Paraît-il que les enfants grandissant avec plusieurs langues sont plus vifs ? En relisant ma chronique, je ne peux que constater la véracité du propos. Ahlalala ! Je vous vois déjà tousser cher Professeur Motte devant ce manque flagrant de modestie. Détrompez-vous, voici une dose d'ironie bien française, omniprésente dans l'œuvre du Docteure Salvayre. Quelle dextérité dans le maniement de l'ironie, que ce soit dans les premiers livres, comme dans La Vie commune, où une vieille chef de publicité souffre de l'arrivée d'une nouvelle secrétaire au bureau, au point d'en dresser un portrait démoniaque, ou dans $B W$, le récit de la vie d'un homme que la Docteure Salvayre connaît trop pour ne pas l'abîmer. Toujours avec humour et amour. Mais nous y reviendrons.

La moitié de ses personnages sont un peu, ou beaucoup, désorientés. Des pommés, des perdus, des frappés du crâne, souvent dans l'adversité. Elle a travaillé plus de cinq ans, de nuit, dans une clinique psychiatrique. Et je me questionne, est-ce qu'un psychiatre est aussi malade que ces patients? A-t-elle trop pratiqué au point de se contaminer ? Faut-il être fou pour écrire? Pour passer des heures à raconter sa vie ou celles des autres? Pourquoi Docteure Salvayre? Pourquoi écrivez-vous ? Elle me donnera la réponse un vendredi soir à dix-huit heures, du fin fond de sa campagne, alors que mes voisins allemands m'attendent pour boire un Glühwein de Noël.

« Une colère cher ami. Une grande colère. J'étais jeune. Invitée dans une soirée chez des bourgeois. Je pensais y 
apprendre des choses, en tirer enseignement. Un grand type m'a dévisagé en me décrivant comme "cette fille qui fait bien modeste". Tu vois à quoi cela tient. Il m'a piquée et j'ai voulu, je ne sais pas ce que j'ai voulu. Mais je crois que tout a démarré là ».

La pulsion. La pulsion de vivre. Docteure Salvayre porte la plume dans la plaie, dans ses plaies, celles du monde, dans ses déchirures, ses meilleurs livres sont écrits avec le cœur fendu, avec la hâte de vouloir le réparer. Un soir, dans le sud de la France, en croquant une crevette, pendant un festival littéraire, alors qu'elle m'écoutait avec une patience de médecin, soudain, elle me fait remarquer que les grands écrivains ont un problème de père, me citant Mann et d'autres. Moi j'ai cherché son père dans ses livres, la mère est là, le père est amoché, plus tard, elle me confiera qu'avec le padre la vie était dure.

À présent, je voudrais aborder un point essentiel de l'écriture de la Docteure Salvayre. Son humanisme. Ses personnages ne sont ni gentils, ni méchants, ni bons, ni salauds. Ils vivent avec défauts, colères, paradoxes. La Docteure Salvayre sait lire. Sait lire dans l'humain, dans la passion et dans la raison, elle nous raconte Bernanos découvrant les horreurs de la guerre d'Espagne, elle prend un camp, celui de l'âme humaine dépassée face à la grande Histoire. Dans La Puissance des mouches, encore un homme, que nous autres humains sans éclat présumons fou, discourt sur son crime. Sur ses raisons. Sur sa trajectoire de vie. La Docteure excelle dans le tête-à-tête, elle triture, et fouille, regarde, ausculte, détaille, nous permet d'établir le diagnostic. Il y a de la compassion. Une immense compassion, quand elle expose les traumatismes et les souffrances de ses héros. Pendant des 
années, dans son bureau, elle a écouté ses patients. Elle s'est interrogée pour les soigner, a accumulé les parcours dans son Hippocampe pour les transformer en une matière première de son œuvre. Elle a aidé ses patients à retrouver leur pleine joie de vivre, l'envie de continuer, leur liberté.

La liberté. Venons-y. Elle est partout, transpire dans chaque livre. Les héros veulent vivre. Et elle rend hommage à leur envie d'embrasser cette liberté. Elle transpire aussi dans les thèmes, et notamment dans ce regard féminin libre et sans concession sur les hommes s'emparant des femmes, de leur sexe. D'ailleurs chez la Docteure Salvayre, je lis un féminisme revendiqué. J'en suis heureux. Elle défend les femmes sans jurer à la fin des hommes. Elle leur donne une voix. Elle pose le masculin sur la balance, laisse l'aiguille monter, ficelle le sachet, le livre au lecteur avec une immense indépendance. Elle n'a pas peur. Elle n'a pas de complexes. Elle est médecin. Et croyez-moi, Professeur Notte, j'ai quelques médecins du côté de ma belle-famille, quatre exactement, et on se marre bien. Aucun complexe, ils savent se rire de tout, d'eux-mêmes, de la vie et des patients. Pour un bon médecin, la vie est tout et elle n'est rien. Elle n'a pas de prix. La vie est le métronome. La vérité. Et pour y survivre, la légèreté est de rigueur. Il est de notoriété publique, que nos médecins français, pendant leur formation à l'internat, se prêtent à toute une série de jeux puérils dignes d'enfants sadiques. Les Français nomment cela l'esprit cabotin, et nous excusons nos bons docteurs, parce qu'ils nous soignent, et aussi parce qu'ils peuvent devenir de bons écrivains. Le métier demande rigueur et précision, chez la Docteure Salvayre, l'écriture témoigne de ces qualités. La psychiatre lit dans la douleur pour dessiner la vérité. Avec l'humilité de ceux qui ont accédé au grand monde 
par le seul mérite de leurs études. Lydie vient d'un milieu populaire, de parents ayant fui la guerre d'Espagne réfugiés en France. C'est un directeur de cours complémentaire, une école pour jeune fille se destinant à être femme au foyer, qui la repérera et la poussera à aller au lycée. La suite, nous la connaissons.

Continuons. Le Petit Traité d'éducation lubrique. Voilà un autre petit livre parfumé de liberté qui raconte l'intimité avec drôlerie. Au moment de sa republication il y a deux ans, j'ai entendu, autour de moi, surtout de la part de jeunes critiques littéraires, des mots d'incompréhension face à ce livre. Professeur Motte, vous vous rendez compte, des jeunes de trente ans, étonnés, pardon, choqués, interdits, ahuris, de découvrir le propos de ce petit livre. Et nous qui pensions que l'époque était à la liberté sexuelle ? L'est-elle vraiment ? Ce n'est pas à moi de savoir, ni de le dire, mais Lydie vous répondrait que oui, qu'avant, on était plus libres, qu'on faisait plus en disant moins, et c'est ce que j'ai cru lire à travers ses lignes. Notamment quand elle raconte l'adolescence espagnole de sa mère.

Venons-y. L'Espagne. Inutile de vous dire que la Docteure Salvayre est malade d'Espagne. De sa langue. Ses histoires sont peuplées de Don Quichotte, un coup, c'est un père, l'autre, c'est une femme de ménage, ou un gardien d'immeuble, des personnages méditerranéens tantôt loufoques, toujours colériques, passionnés, emportés, comme dans sa famille, je crois savoir. Lydie Salvayre semble nous dire que tout comme nous portons le patrimoine génétique de nos parents, nous portons également le patrimoine mémoriel de notre famille. 
Et si je peux me permettre de parler de mon expérience, je comprends cette passion. Je suis né 39 ans après la Docteure Salvayre, dans la même France mais plus vieille. Dans la mienne, on ne m'a jamais dit franchement, mais suggéré habilement d'oublier ma culture familiale pour épouser celle de la terre d'accueil. Était-ce la même pour la Docteure Salvayre ? Si bien qu'à l'âge de ses premiers faits d'armes littéraires, munis de sa formation de spéléologue de l'âme humaine, elle a voulu rattraper le temps perdu. Boire ce lait maternel. Le célébrer. Le magnifier. Nous donner envie de siroter un Tinto de verano, une San Miguel, un Rioja, un Cava, avec son Bernard, celui de BW sur une terrasse baignée de soleil à Valencia.

Bernard, quel homme et quel amour pour cet homme, vous ne trouvez pas Professeur Motte? Quel homme ne rêverait pas d'être ainsi aimé ? Voir sa vie, ses exploits, son intelligence, son tempérament célébré dans un livre. Le meilleur pour moi. Le plus vif. Le plus fort. Peut-être parce que $B W$ raconte en partie un monde que je connais ? Celui de l'édition parisienne. Peut-être parce que dans chacun de nous, il y a un peu de ce Bernard ombrageux, tenu en laisse pour permettre le processus de civilisation. Il est éditeur. Il la lit. La relit. La corrige. Et elle l'aime de son grand amour. Je dirais, je m'avancerais à dire, que derrière cette grande auteure qu'est Lydie Salvayre, il y a un homme discret au grand cœur, nommé Bernard Wallet. Un homme conscient infoutu de digérer la saloperie du monde, un écorché vif, trimballant sa carcasse à corazón ouvert. Comme la Docteure Salvayre.

Elle se soucie du monde, de l'époque, de la nuit qui pourrait tomber, de l'hiver qui avance, elle m'a murmuré ne pas être entièrement satisfaite de son dernier, Tout homme est 
une nuit, je n'étais pas d'accord, ce n'était pas pour la flatter. Je l'ai trouvé mieux que d'autres. Professeur, vous lirez, vous avez lu ce que la France devient, ce que d'un côté les urbains deviennent et de l'autre les campagnards. Vous lirez ce que nous autres Français, avons aimé et chéri, la campagne et l'esprit campagnard, ont dépéri à cause de l'ignorance, l'oubli, le snobisme des gens des grandes villes. Vous lirez ce monde qui glisse, qui peu à peu a pris le parti de détester le monde qui pense et qui lit. Peut-être à raison, parce que ce monde n'a pas voulu accorder le respect qu'il se doit aux peuples des campagnes.

Un dernier point central, cher Professeur Notte ou Motte ou je ne sais quoi. Ne vous enfuyez pas, et excusez mon arrogance tout à fait française. Comme tout bon converti-j'ai obtenu la nationalité française à l'âge de treize ans-, je suis plus royaliste que le roi. Je serai bref. Un dernier point central, la solitude. Cette impression permanente que ses personnages vivent au milieu d'un océan de voix, d'esprit. Qu'ils crient sans être entendus. De ma lecture vorace de ses livres, je crois comprendre que la grande énigme de son œuvre est la solitude. Que peut faire l'être humain face au monde? C'est la question qu'elle semble nous poser livre après livre.

Voilà cher Professeur Motte, ce que je peux dire de l'écriture de Lydie Salvayre. Vous m'avez lancé un sacré défi, celui de lire l'œuvre complète d'un auteur. Périple littéraire auquel je ne m'étais adonné jusqu'alors. Voici comment plonger en quelqu'un. Le voir évoluer. Serrer sa plume. Affuter sa pensée. Dérouler son âme. Son style m'habite à présent, au point que je ne sais plus écrire autrement. Je vais vous faire une confidence. J'ai connu la Docteure Salvayre sur un plateau de télévision. Elle était dans ma diagonale, je 
portais une veste à motif africain, on s'est échangé un regard, puis deux, puis dix, et immédiatement j'ai été frappé d'un coup de foudre d'amour d'amitié. Tout était dans le regard. Vous connaissez cette sensation? Ah oui ? Avec votre chien? Soit pourquoi pas. Dans les mois suivants, nous avons fait connaissance. Rien à dire, à savoir de plus, je savais déjà tout. Je ne suis pas Professeur de littérature, tout juste auteur d'un roman, certes anobli par l'académie Goncourt, juste un lecteur. Un grand lecteur qui par le hasard de la vie a connu quelques écrivains à un âge relativement jeune. Et je peux vous dire, vous assurer, qu'il n'y a que deux catégories d'auteurs, les mauvais et les bons, que leurs livres racontent autant leurs histoires qu'ils décrivent leur intelligence littéraire. L'auteur et son œuvre font corps. Regardez Lydie. Arrêtez-vous un instant. C'est ce que j'ai fait la première fois. Précise, profonde, douce et dure car vraie, toujours bienveillante. Je n'ai rien appris de plus dans ses livres. Lydie est dans la vie comme dans ses livres. Elle ne ment pas. Ne triche pas. Lydie c'est la vérité. 